\title{
Relating Sequent Calculi for Bi-intuitionistic Propositional Logic
}

\author{
Luís Pinto \\ Centro de Matemática, Universidade do Minho, \\ Campus de Gualtar, P-4710-057 Braga, Portugal \\ luis@math.uminho.pt
}

\author{
Tarmo Uustalu \\ Institute of Cybernetics at Tallinn University of Technology, \\ Akadeemia tee 21, EE-12618 Tallinn, Estonia \\ tarmo@cs.ioc.ee
}

\begin{abstract}
Bi-intuitionistic logic is the conservative extension of intuitionistic logic with a connective dual to implication. It is sometimes presented as a symmetric constructive subsystem of classical logic.

In this paper, we compare three sequent calculi for bi-intuitionistic propositional logic: (1) a basic standard-style sequent calculus that restricts the premises of implication-right and exclusion-left inferences to be single-conclusion resp. single-assumption and is incomplete without the cut rule, (2) the calculus with nested sequents by Gore et al., where a complete class of cuts is encapsulated into special "unnest" rules and (3) a cut-free labelled sequent calculus derived from the Kripke semantics of the logic. We show that these calculi can be translated into each other and discuss the ineliminable cuts of the standard-style sequent calculus.
\end{abstract}

\section{Introduction}

Classical logic is a logic of dualities, some of which are made evident with the help of its sequent calculus formulations. For example, the duality between conjunction and disjunction is manifest in the traditional sequent calculus rules for these connectives, or the process of proving a formula $A$, i.e., deriving the sequent $\vdash A$, is dual to the process of refuting $A$, i.e., deriving the sequent $A \vdash$. However, if one considers implication as a "first-class" connective and wants to retain dualities, its dual connective of exclusion (or pseudo-difference, or subtraction) should also have a similar status. In classical logic, exclusion is definable from other connectives: $A \prec B$ is equivalent to $A \wedge \neg B$. Classical logic with exclusion in sequent calculus format has been considered by Curien and Herbelin and Crolard [6, 4], in connection to the study of dualities in functional computation, e.g. the duality of values and continuations or the duality of call-by-value and call-by-name evaluation strategies.

Bi-intuitionistic logic (also known as Heyting-Brouwer logic and subtractive logic) results from classical logic with exclusion by taking the implication to be intuitionistic and, in order to preserve duality, making exclusion dual-intuitionistic (hence the word 'bi'-intuitionistic). Bi-intuitionistic logic can also be seen as the union of intuitionistic logic (lacking exclusion) with dual-intuitionistic logic (lacking implication). The former has the disjunction property while the latter has the dual conjunction property (if $A \wedge B$ is refutable, either $A$ is refutable or $B$ is refutable). In the union, both of these properties are lost, yet one cannot prove excluded middle (nor refute contradiction for the dual-intuitionistic weak negation).

Bi-intuitionistic logic first got attention by C. Rauszer [14, 13, 15], who studied its algebraic and Kripke semantics, alongside with an Hilbert-style system and a sequent calculus. A sequent calculus characterization of the logic is easily obtained by extending the multiple-conclusion sequent calculus for intuitionistic logic à la Dragalin with exclusion rules dual to the implication rules. But contrary to what is suggested, e.g., in [17], cut is not fully eliminable in this calculus. Neither is cut eliminable in the sequent calculus of Rauszer [13] (the proof in the paper is incorrect). 
The lack of cut elimination in a sequent calculus is problematic for proof search, since the subformula property is not guaranteed. In order to overcome this issue, extended sequent calculi have been considered. In particular, a calculus of nested sequents was proposed by Goré, Postniece and Tiu [7] and a calculus with labelled formulas was proposed by the authors [11].

In the presence of various sequent calculi for bi-intuitionistic logic, a natural question to ask is how they relate to each other. In this paper, we give translations between the standard-style (=Dragalin-style), nested and labelled sequent calculi for bi-intuitionistic propositional logic. By analysing such translations, we are able to identify a complete class of cuts for the formulation with standard sequents and we are able to read the proofs resulting from proof search in the nested or labelled sequent calculus back into the standard-style sequent calculus. Reed and Pfenning [16] have remarked that relating labelled intuitionistic derivations with standard unlabelled ones is "a surprisingly difficult question".

\section{The syntax and semantics of BiInt}

We start by defining the syntax and semantics of bi-intuitionistic propositional logic (BiInt).

The language of BiInt extends that of intuitionistic propositional logic (Int), by one connective, exclusion, thus the formulas are given by the grammar:

$$
A, B:=p|\top| \perp|A \wedge B| A \vee B|A \supset B| A \prec B
$$

where $p$ ranges over a denumerable set of propositional variables which give us atoms; the formula $A \prec B$ is the exclusion of $B$ from $A$. We do not take negations as primitive, but in addition to the intuitionistic (or strong) negation, there is also a dual-intuitionistic (or weak) negation. The two negations are definable by $\neg A:=A \supset \perp$ and $\backsim A:=\top \prec A$.

The semantics of BiInt is usually given à la Kripke, although one can also proceed from an algebraic semantics (in terms of Heyting-Brouwer algebras) and there are further alternatives. The Kripke semantics is about truth relative to worlds in Kripke structures that are the same as for Int. A Kripke structure is a triple $K=(W, \leq, I)$ where $W$ is a non-empty set whose elements we think of as worlds, $\leq$ is a preorder (reflexive-transitive binary relation) on $W$ (the accessibility relation) and $I$ - the interpretation-is an assignment of sets of propositional variables to the worlds, which is monotone w.r.t. $\leq$, i.e., whenever $w \leq w^{\prime}$, we have $I(w) \subseteq I\left(w^{\prime}\right)$.

Truth in Kripke structures is defined as for Int, but covers also exclusion, interpreted dually to implication as possibility in the past:

- $w \models p$ iff $p \in I(w)$;

- $w \models \top$ always; $w \models \perp$ never;

- $w \models A \wedge B$ iff $w \models A$ and $w \models B ; w \models A \vee B$ iff $w \models A$ or $w \models B$;

- $w \models A \supset B$ iff, for any $w^{\prime} \geq w, w^{\prime} \not \models A$ or $w^{\prime} \models B$;

- $w \models A \prec B$ iff, for some $w^{\prime} \leq w, w^{\prime} \models A$ and $w^{\prime} \not \models B$.

A formula is called valid if it is true in all worlds of all structures. It is easy to see that monotonicity extends from atoms to all formulas thanks to the universal and existential semantics of implication and exclusion.

It is important for this paper that instead of general Kripke structures, one may equivalently work with Kripke trees. These are Kripke structures $(W, \leq, I)$ where $W$ is finite and the preorder $\leq$ arises as the reflexive-transitive closure of some binary relation $\rightarrow$ on $W$, subject to the condition that any two 
Initial rule and cut (necessary):

$$
\overline{\Gamma, A \vdash A, \Delta} \text { hyp } \quad \frac{\Gamma \vdash A, \Delta \quad \Gamma, A \vdash \Delta}{\Gamma \vdash \Delta} c u t
$$

Structural rules:

$$
\frac{\Gamma \vdash \Delta}{\Gamma, A \vdash \Delta} \text { weakL } \quad \frac{\Gamma \vdash \Delta}{\Gamma \vdash A, \Delta} \text { weakR } \quad \frac{\Gamma, A, A \vdash \Delta}{\Gamma, A \vdash \Delta} \operatorname{contrL} \quad \frac{\Gamma \vdash A, A, \Delta}{\Gamma \vdash A, \Delta} \text { contrR }
$$

\section{Logical rules:}

$$
\begin{array}{ccc}
\frac{\Gamma \vdash \Delta}{\Gamma, \top \vdash \Delta} \top L & \frac{\Gamma, A, B \vdash \Delta}{\Gamma \vdash \top, \Delta} \top R \quad \frac{\Gamma \vdash A, \Delta \quad \Gamma \vdash B, \Delta}{\Gamma, A \wedge B \vdash \Delta} \wedge R \quad \frac{\Gamma \vdash A \wedge B, \Delta}{\Gamma, A \vdash \Delta \quad \Gamma, B \vdash \Delta} \\
\frac{\Gamma \vdash \Delta}{\Gamma, \perp \vdash \Delta} \perp L & \frac{\Gamma \vdash A}{\Gamma \vdash \perp, \Delta} \perp R \quad \frac{\Gamma \vdash A, B, \Delta}{\Gamma \vdash A \vee B, \Delta} \vee R \\
\frac{\Gamma, A \supset B \vdash A, \Delta \quad \Gamma, B \vdash \Delta}{\Gamma, A \supset B \vdash \Delta} \supset L \quad \frac{\Gamma, A \vdash B}{\Gamma \vdash A \supset B, \Delta} \supset R \\
\frac{A \vdash B, \Delta}{\Gamma, A \prec B \vdash \Delta} \prec L \quad \frac{\Gamma \vdash A, \Delta \quad \Gamma, B \vdash A \prec B, \Delta}{\Gamma \vdash A \prec B, \Delta} \prec R
\end{array}
$$

Figure 1: Inference rules of $\mathbf{L B i I}$

worlds $w, w^{\prime}$ are related by the reflexive-transitive-symmetric closure of $\rightarrow$ in a unique way $\left(w^{\prime}\right.$ is reached from $w$ by exactly one path along $\rightarrow \cup \leftarrow$ ).

It is also a basic observation that the Gödel translation of Int into the modal logic $\mathbf{S 4}$ extends to a translation into the future-past tense logic KtT4 (cf. [8]). As the semantics of KtT4 does not enforce monotonicity of interpretations, atoms must be translated as future necessities or past possibilities (these are always monotone): $p^{\#}=\square p$ (or $\left.\diamond\right) ; \top^{\#}=\top ; \perp^{\#}=\perp ;(A \wedge B)^{\#}=A^{\#} \wedge B^{\#} ;(A \vee B)^{\#}=A^{\#} \vee B^{\#}$; $(A \supset B)^{\#}=\square\left(A^{\#} \supset B^{\#}\right) ;(A \prec B)^{\#}=\triangleleft\left(A^{\#} \prec B^{\#}\right)$.

\section{Three sequent calculi for BiInt}

We will now recall three different sequent calculi for BiInt that we will later compare to each other.

\subsection{Standard-style sequent calculus LBiI}

A sequent calculus for BiInt is most easily obtained by extending Dragalin's sequent calculus for Int, as has been done by Restall [17] and Crolard [4]. (Rauszer's [13] original sequent calculus was different.) In Dragalin's system, sequents are multiple-conclusion, but the $\supset R$ rule is constrained. The extension, which we will now show, imposes a dual constraint on the $\prec L$ rule.

The sequents of our calculus (henceforth referred to as the standard-style calculus LBiI) are pairs $\Gamma \vdash \Delta$ where $\Gamma, \Delta$ (the antecedent and succedent) are finite multisets of formulas (we omit braces and denote union by comma as usual). The inference rules of $\mathbf{L B i I}$ are displayed in Fig. 1. 
Note that $\Delta$ is missing in the premise of the $\supset R$ rule and dually in the premise of $\prec L$ we do not have the context $\Gamma$.

Regarding structural rules, both in LBiI and the other two sequent calculi (N-LBiI and L-LBiI) considered in this paper, we have chosen to work with formulations oriented at root-first proof search, which means that, as a general guideline, we want to have our inference rules "as invertible as possible". We have weakening and contraction built in to the other rules to the degree that LBiI and L-LBiI are complete without explicit versions of them. This requires of course that the two-premise rules are context-sharing etc. But there are also more specific consequences. In LBiI, we have duplications of the implication and exclusion formulas in the premises of the $\supset L$ and $\prec R$ rules.

LBiI is sound and complete for the Kripke semantics of BiInt for the following generalization of validity from formulas to sequents. A sequent $\Gamma \vdash \Delta$ is taken to be valid if, for any in Kripke structure $(W, \leq, I)$ and any world $w$, we have that if all formulas in $\Gamma$ are true in $w$, then so is some formula in $\Delta$. This has been proved (for variants of LBiI), e.g., by Restall [17] and Monteiro [9].

However, LBiI is incomplete without cut, as shown by Pinto and Uustalu in 2003 (private email message from T. Uustalu to R. Goré, 13 Sept. 2004, quoted in [3]). It suffices to consider the obviously valid sequent $p \vdash q, r \supset((p \prec q) \wedge r)$. The only possible last inference (other than weakening and contraction, which are redundant) in a derivation could be

$$
\frac{p, r \vdash(\stackrel{?}{p} \prec q) \wedge r}{p \vdash q, r \supset((p \prec q) \wedge r)} \supset R
$$

but the premise is invalid as the succedent formula $q$ has been lost. With cut, the sequent can be proved as follows:

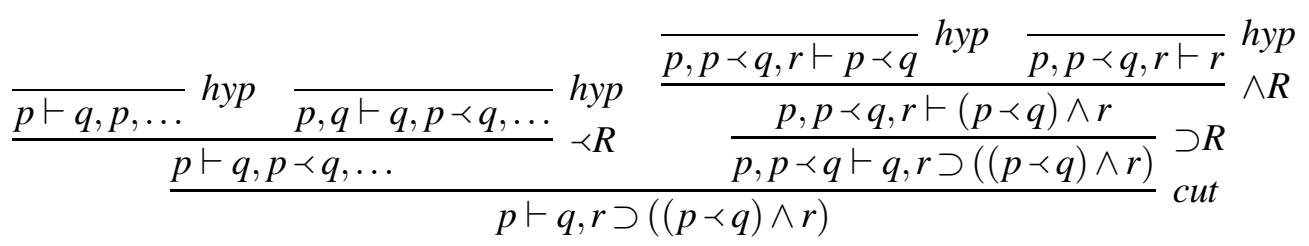

Notice that permutation of the cut on the exclusion $p \prec q$ up past the $\supset R$ inference, for which the cut formula is a side formula, is not possible. This is one type of cuts that cannot be eliminated; there are altogether three such types [9]. This situation reminds of the naive standard-style sequent calculus for S5 where the sequent $p \vdash \square \diamond p$ cannot be proved without cut, but can be proved by applying cut to the sequents $p \vdash \diamond p$ and $\diamond p \vdash \square \diamond p$ that are provable without cut.

In Sec. 5, with the help of the translations proposed in this paper, we identify a class of cuts complete for $\mathbf{L B i I}$.

\subsection{Nested sequent calculus N-LBiI}

Next we introduce a calculus $\mathbf{N}-\mathbf{L B i I}$ of nested sequents, which is a minor variation of the calculus LBiInt $_{1}$ of Goré et al. [7] 1 N-LBiI is an extension of $\mathbf{~} \mathbf{~ B i I}$ where the concept of contexts is generalized so that, alongside formulas, they can also contain nested sequents, manipulated by dedicated additional inference rules.

\footnotetext{
${ }^{1}$ The main difference is that LBiInt $_{1}$ does not build weakening and contraction into other rules, as we do in some cases to have a direct match with corresponding rules in the other systems considered.
} 


\section{Rules for nested sequents:}

$$
\begin{array}{ll}
\frac{\Gamma_{0} \vdash \Delta_{0}, \Delta}{\Gamma,\left(\Gamma_{0} \vdash \Delta_{0}\right) \vdash \Delta} \text { nestL } & \frac{\Gamma, \Gamma_{0} \vdash \Delta_{0}}{\Gamma \vdash\left(\Gamma_{0} \vdash \Delta_{0}\right), \Delta} \text { nestR } \\
\frac{\Gamma,\left(\Gamma_{0} \vdash \Delta_{0}\right) \vdash \Delta}{\Gamma, \Gamma_{0} \vdash \Delta_{0}, \Delta} \text { unnestL } & \frac{\Gamma \vdash\left(\Gamma_{0} \vdash \Delta_{0}\right), \Delta}{\Gamma, \Gamma_{0} \vdash \Delta_{0}, \Delta} \text { unnestR }
\end{array}
$$

Figure 2: Inference rules of $\mathbf{N}-\mathbf{L B i I}$ for manipulating nested sequents; differently from $\mathbf{L B i I}$, cut is redundant

The sequents of N-LBiI (ranged over by $S$ ) are defined simultaneously with contexts (ranged over by $\Gamma, \Delta)$ by the following grammar:

$$
\begin{aligned}
S & ::=\Gamma \vdash \Delta \\
\Gamma, \Delta & ::=\emptyset|A, \Gamma| S, \Gamma
\end{aligned}
$$

where contexts, just as in LBiI, are quotiented down to multisets (so identified up to permutations of the member formulas/nested sequents). Just as commas in antecedents and succedents intuitively correspond to conjunctions and disjunctions, nested turnstiles should be understood as structural-level implications and exclusions 2

The inference rules of $\mathbf{N}-\mathbf{L B i I}$ are those of $\mathbf{L B i I}$ in Fig. 1) (including the cut rule and the structural rules) together with additional inference rules for introducing and eliminating nested sequents. These additional rules appear in Fig. 2. The nest $L$ and nest $R$ rules are structural versions of $\prec L$ and $\supset R$. The unnest $L / R$ rules are elimination rules for exclusions on the left and implications on the right. It is fair to think of them as masqueraded versions of certain rather specific types of cuts (we come to this in Sec. 5). 3

Stating soundness and completeness of N-LBiI requires defining what it means for a nested sequent to be valid. This is achieved via a translation that "flattens" nested sequents into standard sequents, reducing validity of nested sequents to that of standard sequents. We give a formal definition of this translation of sequents in Sec. 4.1, where we show that derivations of N-LBiI can be translated into LBiI. Goré et al. [7] established soundness of N-LBiI wrt. this notion of validity directly, but showed completeness by an embedding of Rauszer's sequent calculus [13].

They also showed cut to be redundant in the strong sense of existence of a cut-eliminating transformation of derivations [7]. The example of the previous section is proved in N-LBiI without cut (but with unnest $L$ ) as follows:

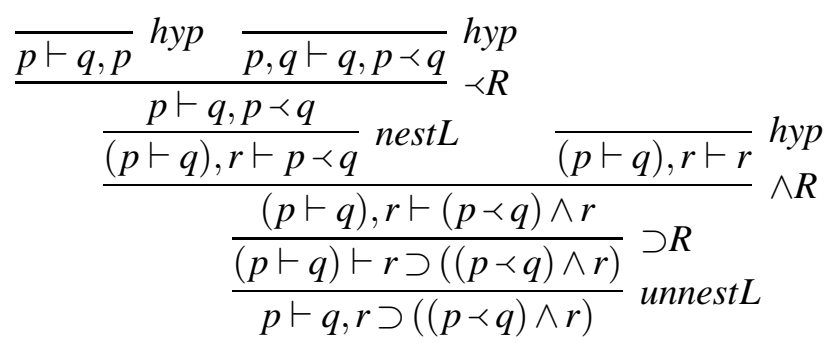

\footnotetext{
${ }^{2}$ In [7], a nested sequent in the antecedent (resp. succedent) of a parent sequent (a structural-level exclusion resp. implication) is written $\Gamma<\Delta$ (resp. $\Gamma>\Delta)$.

${ }^{3}$ For the sake of simplicity of presentation, we have opted for a formulation of unnest $L / R$ rules that does not incorporate formula contraction. For a version of $\mathbf{N}-\mathbf{L B i I}$ that is complete without the $\operatorname{contr} L / R$ rules, the unnest $L / R$ rules must be stated differently.
} 


\subsection{Labelled sequent calculus L-LBiI}

The third sequent calculus we consider in this paper is a labelled sequent calculus L-LBiI, a variation on the calculus L of ours [11] 4 The design of L-LBiI follows the method of S. Negri [10] for obtaining cutfree sequent calculi for normal modal logics defined by frame conditions of a certain type. Essentially, L-LBiI is a formalization of the first-order theory of the Kripke semantics of BiInt, using an explicit device of labels for worlds.

A sequent of $\mathbf{L}-\mathbf{L B i I}$ is a triple $\Gamma \vdash_{G} \Delta$ where $G$ is a label tree and $\Gamma$ and $\Delta$ are labelled contexts. More precisely, the label tree $G=(N, E)$ is a directed graph that has its set of nodes $N$ (called labels) nonempty and finite and is an undirected tree in the sense that any nodes $x, y$ are in the relation $\left(E \cup E^{-1}\right)^{*}$ in only one way, i.e., are connected by a single path of forward and backward arcs. We write $|G|$ for $N$ and $x G y$ for $(x, y) \in E$. The labelled contexts $\Gamma$ and $\Delta$ are multisets of labelled formulas and these, in their turn, are pairs $x$ : $A$ with $x$ a label drawn from $|G|$ and $A$ a formula.

Any label tree can be built (generally in many ways) from constructions $\langle x\rangle$ for the tree with a single node $x,(x, y)$ for the tree with two nodes $x, y$ and one $\operatorname{arc} x G y$, and $G \oplus_{x} G^{\prime}$ for the join of two trees at $x$. In the last construction, we require (as a welldefinedness condition) that the trees $G$ and $G^{\prime}$ satisfy $|G| \cap\left|G^{\prime}\right|=\{x\}$, which guarantees that the joint graph really is a tree. Conversely, any directed graph built in terms of these constructions is necessarily a tree.

Intuitively, we use label trees to represent Kripke trees and a labelled formula is about truth at a particular world.

The inference rules of L-LBiI are presented in Fig. 3. Some of them have provisos, that we also write as rule premises. The conditions $G \downarrow y$ resp. $G \uparrow y$ mean that there is no $z$ such that $z G y$ resp. $y G z$. The wellformedness condition of any $\oplus$ expression occurrence must also be read as a proviso. In the rules $\supset R$ and $\prec L$, we have freshness conditions on $y$. Note the presence of the monotonicity rules intuitively accounting for propagation of truth (resp. falsity) to future (resp. past) worlds. The nodesplit and nodemerge structural rules are auxiliary and redundant to the degree of existence of eliminating transformations (alongside weak and contr); we included them here, because they come handy in the translation of Sec. 4.3. nodesplit $L / R$ split a node into a pair of nodes connected by an arc, so that no paths are lost. nodemerge $L / R$ merge two nodes connected by an arc.

A labelled sequent $\Gamma \vdash_{G} \Delta$ is valid if, for any Kripke structure $(W, \leq, I)$ and function $v:|G| \rightarrow W$ such that $x G y$ implies $v(x) \leq v(y)$, we have that, if $v(x) \models A$ for every $x: A$ in $\Gamma$, then $v(x) \models A$ for some $x: A$ in $\Delta$. L-LBiI is sound and complete wrt. this notion of validity.

L-LBiI is complete without cut (as we proved by a semantic argument in [11]) and one should also be able to give a cut-eliminating transformation of derivations.

Our counterexample to cut elimination in $\mathbf{L B i I}$ is proved in $\mathbf{L - L B i I}$ as follows:

$$
\begin{aligned}
& \frac{\overline{x: p, \ldots \vdash_{(x, y)} x: p, \ldots} \text { hyp } \overline{x: q, \ldots \vdash_{(x, y)} x: q, \ldots}}{x: p, \ldots \vdash_{(x, y)} x: q, x: p \prec q, \ldots} \prec R \\
& \frac{x: p, \ldots \vdash_{(x, y)} x: q, x: p \prec q, \ldots}{x: p, \ldots \vdash_{(x, y)} x: q, y: p \prec q} \text { monot } \quad \frac{}{x: p, y: r \vdash_{(x, y)} x: q, y: r} \text { hyp } \\
& \frac{x: p, y: r \vdash_{(x, y)} x: q, y:(p \prec q) \wedge r}{x: p \vdash_{\langle x\rangle} x: q, x: r \supset((p \prec q) \wedge r)} \supset R
\end{aligned}
$$

\footnotetext{
${ }^{4}$ In fact, L-LBiI lies between the calculi $\mathrm{L}$ and $\mathrm{L}^{*}$ of [11]. Similarly to $\mathrm{L}^{*}$, the sequent calculus here is a calculus of finite Kripke trees rather than general Kripke structures, so we reason in terms of adjacency $\rightarrow$ rather than the induced accessibility relation $\leq=\rightarrow^{*}$. Differently from $\mathrm{L}$, there are no reflexivity and transitivity rules, the monotonicity rules propagate truth/falsity to adjacent labels only (but can, of course, be applied multiple times), and the $\supset L$ and $\prec R$ rules analyse and duplicate the main formula locally.
} 
Initial rule and cut (redundant):

$$
\frac{\Gamma, x: A \vdash_{G} x: A, \Delta}{\Gamma y p} \quad \frac{\Gamma \vdash_{G} x: A, \Delta \quad \Gamma, x: A \vdash_{G} \Delta}{\Gamma \vdash_{G} \Delta} \text { cut }
$$

\section{Structural rules:}

$$
\begin{aligned}
\frac{\Gamma \vdash_{G} \Delta}{\Gamma, x: A \vdash_{G} \Delta} \text { weakL } & \frac{\Gamma \vdash_{G} \Delta}{\Gamma \vdash_{G} x: A, \Delta} \text { weakR } \\
\frac{\Gamma, x: A, x: A \vdash_{G} \Delta}{\Gamma, x: A \vdash_{G} \Delta} \text { contrL } & \frac{\Gamma \vdash_{G} x: A, x: A, \Delta}{\Gamma \vdash_{G} x: A, \Delta} \text { contrR } \\
\frac{G \downarrow x \quad \Gamma \vdash_{G_{0} \oplus_{y} G[y / x]} \Delta}{\Gamma \vdash_{G_{0} \oplus_{y}(y, x) \oplus_{x} G} \Delta} \text { nodesplitU } & \frac{G \uparrow x \quad \Gamma \vdash_{G[y / x] \oplus_{y} G_{0}} \Delta}{\Gamma \vdash_{G \oplus_{x}(x, y) \oplus_{y} G_{0}} \Delta} \text { nodesplitD } \\
\frac{\Gamma \vdash_{G_{0} \oplus_{y}(y, x) \oplus_{x} G} \Delta}{\Gamma[x / y] \vdash_{G_{0}[x / y] \oplus_{x} G} \Delta[x / y]} \text { nodemergeD } & \frac{\Gamma \vdash_{G \oplus_{x}(x, y) \oplus_{y} G_{0}} \Delta}{\Gamma[x / y] \vdash_{G \oplus_{x} G_{0}[x / y]} \Delta[x / y]} \text { nodemergeU }
\end{aligned}
$$

\section{Monotonicity rules:}

$$
\frac{x G y \quad \Gamma, x: A, y: A \vdash_{G} \Delta}{\Gamma, x: A \vdash_{G} \Delta} \text { monot } L \quad \frac{y G x \quad \Gamma \vdash_{G} y: A, x: A, \Delta}{\Gamma \vdash_{G} x: A, \Delta} \text { monotR }
$$

\section{Logical rules:}

$$
\begin{aligned}
& \frac{\Gamma \vdash_{G} \Delta}{\Gamma, x: \top \vdash_{G} \Delta} \top L \quad \overline{\Gamma \vdash_{G} x: \top, \Delta} \top R \\
& \frac{\Gamma, x: A, x: B \vdash_{G} \Delta}{\Gamma, x: A \wedge B \vdash_{G} \Delta} \wedge L \quad \frac{\Gamma \vdash_{G} x: A, \Delta \quad \Gamma \vdash_{G} x: B, \Delta}{\Gamma \vdash_{G} x: A \wedge B, \Delta} \wedge R \\
& \overline{\Gamma, x: \perp \vdash_{G} \Delta} \perp L \quad \frac{\Gamma \vdash_{G} \Delta}{\Gamma \vdash_{G} x: \perp, \Delta} \perp R \\
& \frac{\Gamma, x: A \vdash_{G} \Delta \quad \Gamma, x: B \vdash_{G} \Delta}{\Gamma, x: A \vee B \vdash_{G} \Delta} \vee L \quad \frac{\Gamma \vdash_{G} x: A, x: B, \Delta}{\Gamma \vdash_{G} x: A \vee B, \Delta} \vee R \\
& \frac{\Gamma, x: A \supset B \vdash_{G} x: A, \Delta \quad \Gamma, x: B \vdash_{G} \Delta}{\Gamma, x: A \supset B \vdash_{G} \Delta} \supset L \quad \frac{\Gamma, y: A \vdash_{G \oplus_{x}(x, y)} y: B, \Delta}{\Gamma \vdash_{G} x: A \supset B, \Delta} \supset R \\
& \frac{\Gamma, y: A \vdash_{(y, x) \oplus_{x} G} y: B, \Delta}{\Gamma, x: A \prec B \vdash_{G} \Delta} \prec L \quad \frac{\Gamma \vdash_{G} x: A, \Delta \quad \Gamma, x: B \vdash_{G} x: A \prec B, \Delta}{\Gamma \vdash_{G} x: A \prec B, \Delta} \prec R
\end{aligned}
$$

Figure 3: Inference rules of $\mathbf{L}-\mathbf{L B i I}$ 
Notice the downward information propagation by the monotR inference to an already existing label.

\section{Translations}

In this section, we study syntactic embeddings between the three calculi.

We present six translations in all possible directions according to the following plan (the sixth translation we will only sketch).

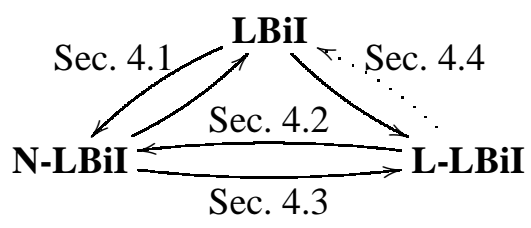

\subsection{From N-LBiI to LBiI and back}

As sequents and rules of $\mathbf{L B i I}$ are also sequents and rules of N-LBiI, a derivation in LBiI is also a derivation in N-LBiI. Note, however, that a cut in LBiI is rendered by a cut also in N-LBiI. This is an issue and we will reconsider it in section Sec. 5 .

For now, we move on to the converse direction.

We define simultaneously two functions on nested contexts $|(-)|^{L}$ and $|(-)|^{R}$ that produce formulas. They are meant to be applied to antecedents and succedents of sequents. We also introduce two further functions $\|(-)\|^{L}$ and $\|\left.(-)\right|^{R}$, defined in terms of $|(-)|^{L}$ and $|(-)|^{R}$, to produce standard contexts instead of formulas. They are used to translate top-level sequents and avoid unnecessary rewriting of commas as $\wedge$ or $\vee$.

$$
\begin{aligned}
|\emptyset|^{L} & =\top & |\emptyset|^{R} & =\perp \\
|A, \Gamma|^{L} & =A \wedge|\Gamma|^{L} & |A, \Gamma|^{R} & =A \vee|\Gamma|^{R} \\
\left|\left(\Gamma_{0} \vdash \Delta_{0}\right), \Gamma\right|^{L} & =\left(\left|\Gamma_{0}\right|^{L} \prec\left|\Delta_{0}\right|^{R}\right) \wedge|\Gamma|^{L} & \left|\left(\Gamma_{0} \vdash \Delta_{0}\right), \Gamma\right|^{R} & =\left(\left|\Gamma_{0}\right|^{L} \supset\left|\Delta_{0}\right|^{R}\right) \vee|\Gamma|^{R} \\
\|\emptyset\|^{L} & =\emptyset & \|\emptyset\|^{R} & =\emptyset \\
\|A, \Gamma\|^{L} & =A,\|\Gamma\|^{L} & \|A, \Gamma\|^{R} & =A,\|\Gamma\|^{R} \\
\left\|\left(\Gamma_{0} \vdash \Delta_{0}\right), \Gamma\right\|^{L} & =\left(\left|\Gamma_{0}\right|^{L} \prec\left|\Delta_{0}\right|^{R}\right),\|\Gamma\|^{L} & \left\|\left(\Gamma_{0} \vdash \Delta_{0}\right), \Gamma\right\|^{R} & =\left(\left|\Gamma_{0}\right|^{L} \supset\left|\Delta_{0}\right|^{R}\right),\|\Gamma\|^{R}
\end{aligned}
$$

Theorem 1 If $\Gamma \vdash \Delta$ is derivable in $\mathbf{N}$-LBiI, then $\|\Gamma\|^{L} \vdash\|\Delta\|^{R}$ is derivable in $\mathbf{L B i I}$.

Proof The proof is by induction on the structure of the $\mathbf{N}$-LBiI derivation of $\Gamma \vdash \Delta$. The cases corresponding to rules other than the nesting rules are immediate, since there is a directly matching rule in LBiI.

Case nestR: The given derivation has the form

$$
\begin{gathered}
\vdots \pi \\
\frac{\Gamma, \Gamma_{0} \vdash \Delta_{0}}{\Gamma \vdash\left(\Gamma_{0} \vdash \Delta_{0}\right), \Delta} \text { nest } R
\end{gathered}
$$

It can be mapped to

$$
\begin{gathered}
\vdots \text { IH on } \pi \\
\frac{\|\Gamma\|^{L},\left\|\Gamma_{0}\right\|^{L} \vdash\left\|\Delta_{0}\right\|^{R}}{\frac{\|\Gamma\|^{L},\left|\Gamma_{0}\right|^{L} \vdash\left|\Delta_{0}\right|^{R}}{\|\Gamma\|^{L} \vdash\left|\Gamma_{0}\right|^{L} \supset\left|\Delta_{0}\right|^{R},\|\Delta\|^{R}} \supset R}(\wedge R R)^{*}
\end{gathered}
$$


Case unnest $L$ : The given derivation is of the form

$$
\begin{gathered}
\vdots \\
\frac{\Gamma,\left(\Gamma_{0} \vdash \Delta_{0}\right) \vdash \Delta}{\Gamma, \Gamma_{0} \vdash \Delta_{0}, \Delta} \text { unnestL }
\end{gathered}
$$

and we can transform it to

$$
\begin{aligned}
& \begin{array}{c}
\vdots \text { IH on } \pi \\
\frac{\|\Gamma\|^{L},\left|\Gamma_{0}\right|^{L} \prec\left|\Delta_{0}\right|^{R} \vdash\|\Delta\|^{R}}{\|\Gamma\|^{L},\left\|\Gamma_{0}\right\|^{L},\left|\Gamma_{0}\right|^{L} \prec\left|\Delta_{0}\right|^{R} \vdash\left\|\Delta_{0}\right\|^{R},\|\Delta\|^{R}}(\text { weakL } / R)^{*}
\end{array}
\end{aligned}
$$

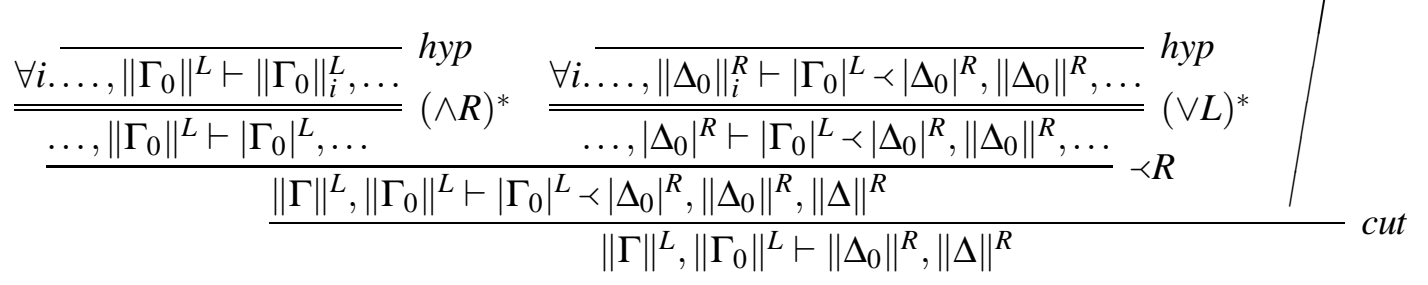

\subsection{From L-LBiI to N-LBiI}

The translation from $\mathbf{L}-\mathbf{L B i I}$ to $\mathbf{N}-\mathbf{L B i I}$ is more involved than those of the previous section, but also more illuminating.

The translation of a labelled sequent into a nested sequent follows the idea that we can view any label of the label tree as its root (intuitively, the focus of attention) and produce a nesting structure for a nested sequent by mimicking this rooted tree.

The translation of a labeled sequent wrt. a chosen label from its label tree is defined by recursion on the rooted tree structure by

$$
\begin{aligned}
& \left\langle\langle\Gamma \vdash\langle x\rangle\langle\rangle\rangle_{x}=\Gamma(x) \vdash \Delta(x)\right. \\
& \left\langle\left\langle\Gamma \vdash_{G \oplus_{x}(x, y) \oplus_{y} G_{0}} \Delta\right\rangle\right\rangle_{x}=\Lambda \vdash\left(\Lambda_{0} \vdash \Pi_{0}\right), \Pi \\
& \text { where } \Lambda \vdash \Pi=\left\langle\left\langle\Gamma[G] \vdash_{G} \Delta[G]\right\rangle\right\rangle_{x} \text { and } \Lambda_{0} \vdash \Pi_{0}=\left\langle\left\langle\Gamma\left[G_{0}\right] \vdash_{G_{0}} \Delta\left[G_{0}\right]\right\rangle\right\rangle_{y} \\
& \left\langle\left\langle\Gamma \vdash \vdash_{G_{0} \oplus_{y}(y, x) \oplus_{x} G} \Delta\right\rangle\right\rangle_{x}=\Lambda,\left(\Lambda_{0} \vdash \Pi_{0}\right) \vdash \Pi \\
& \text { where } \Lambda \vdash \Pi=\left\langle\left\langle\Gamma[G] \vdash_{G} \Delta[G]\right\rangle\right\rangle_{x} \text { and } \Lambda_{0} \vdash \Pi_{0}=\left\langle\left\langle\Gamma\left[G_{0}\right] \vdash_{G_{0}} \Delta\left[G_{0}\right]\right\rangle\right\rangle_{y}
\end{aligned}
$$

where $\Gamma(x)=\{A \mid x: A \in \Gamma\}$ and $\Gamma[G]=\{x: A|x \in| G \mid$ and $x: A \in \Gamma\}$.

Intuitively, the formulas labelled with $x$ in the given sequent are kept where they are, whereas those with labels reachable through the labels immediately below resp. above $x$ are arranged into nested sequent members of the antecedent resp. succedent of the top-level nested sequent produced.

Lemma 1 (Readdressing) For any $z, x \in|G|$, if $\left\langle\left\langle\Gamma \vdash_{G} \Delta\right\rangle\right\rangle_{z}$ is derivable in N-LBiI, then so is $\left\langle\left\langle\Gamma \vdash_{G}\right.\right.$ $\Delta\rangle\rangle_{x}$.

Proof By induction on the unique path along $G \cup G^{-1}$ from $x$ to $z$. The base case $x=z$ is trivial.

We consider one of the two symmetric step cases, namely the one where $x G y$. In this case we have $G=G^{\prime} \oplus_{x}(x, y) \oplus_{y} G_{0}$, with the path from $y$ to $z$ lying in $G_{0}$. 
The given derivation is

$$
\begin{gathered}
\vdots \\
\left\langle\left\langle\Gamma \vdash_{G} \Delta\right\rangle\right\rangle_{z}
\end{gathered}
$$

The nested sequent $\left\langle\left\langle\Gamma \vdash_{G} \Delta\right\rangle\right\rangle_{x}$ can be derived by

$$
\begin{gathered}
\vdots \text { IH on } \pi \\
\frac{\Lambda_{0},(\Lambda \vdash \Pi) \vdash \Pi_{0}}{\frac{(\Lambda \vdash \Pi) \vdash\left(\Lambda_{0} \vdash \Pi_{0}\right)}{\Lambda \vdash\left(\Lambda_{0} \vdash \Pi_{0}\right), \Pi} \text { nest } R} \text { unnestL }
\end{gathered}
$$

where $\Lambda \vdash \Pi=\left\langle\left\langle\Gamma\left[G^{\prime}\right] \vdash_{G^{\prime}} \Delta\left[G^{\prime}\right]\right\rangle\right\rangle_{x}$ and $\Lambda_{0} \vdash \Pi_{0}=\left\langle\left\langle\Gamma\left[G_{0}\right] \vdash_{G_{0}} \Delta\left[G_{0}\right]\right\rangle\right\rangle_{y}$, so that $\left\langle\left\langle\Gamma \vdash_{G} \Delta\right\rangle\right\rangle_{x}=\Lambda \vdash$ $\left(\Lambda_{0} \vdash \Pi_{0}\right), \Pi$ whereas $\left\langle\left\langle\Gamma \vdash_{G} \Delta\right\rangle\right\rangle_{y}=\Lambda_{0},(\Lambda \vdash \Pi) \vdash \Pi_{0}$.

Theorem 2 If $\Gamma \vdash_{G} \Delta$ is derivable in $\mathbf{L}-\mathbf{L B i I}$, then $\left\langle\left\langle\Gamma \vdash_{G} \Delta\right\rangle\right\rangle_{x}$ is derivable in $\mathbf{N}-\mathbf{L B i I}$ for any $x \in|G|$.

Proof By induction on the derivation of $\Gamma \vdash_{G} \Delta$ in $\mathbf{L}-\mathbf{L B i I}$. We show the prototypical cases.

Case monot $L$ : The given derivation is of the form

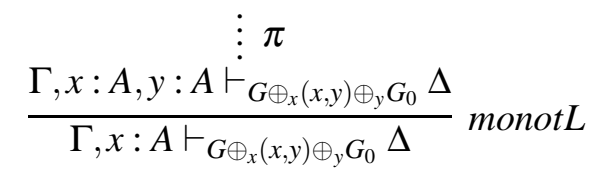

By readdressing, it suffices to prove $\left\langle\left\langle\Gamma, x: A \vdash_{G \oplus_{x}(x, y) \oplus_{y} G_{0}} \Delta\right\rangle\right\rangle_{x}$.

We construct this derivation:

$$
\begin{gathered}
\vdots \text { IH on } \pi, y \\
\frac{(\Lambda, A \vdash \Pi), \Lambda_{0}, A \vdash \Pi_{0}}{\frac{(\Lambda . A \vdash \Pi), A \vdash\left(\Lambda_{0} \vdash \Pi_{0}\right)}{\Lambda, A . A \vdash\left(\Lambda_{0} \vdash \Pi_{0}\right), \Pi} \text { nest } R} \text { unnest } L \\
\Lambda, A \vdash\left(\Lambda_{0} \vdash \Pi_{0}\right), \Pi \\
\text { contrL }
\end{gathered}
$$

Here, $\Lambda \vdash \Pi=\left\langle\left\langle\Gamma[G] \vdash_{G} \Delta[G]\right\rangle\right\rangle_{x}$ and $\Lambda_{0} \vdash \Pi_{0}=\left\langle\left\langle\Gamma\left[G_{0}\right] \vdash_{G_{0}} \Delta\left[G_{0}\right]\right\rangle\right\rangle_{y}$, which gives us $\langle\langle\Gamma, x$ : $\left.\left.A \vdash_{G \oplus_{x}(x, y) \oplus_{y} G_{0}} \Delta\right\rangle\right\rangle_{x}=\Lambda, A \vdash\left(\Lambda_{0} \vdash \Pi_{0}\right), \Pi$ and $\left\langle\left\langle\Gamma, x: A, y: A \vdash_{G \oplus_{x}(x, y) \oplus_{y} G_{0}} \Delta\right\rangle\right\rangle_{y}=(\Lambda, A \vdash \Pi), \Lambda_{0}, A \vdash$ $\Pi_{0}$.

Case $\supset R$ : The given derivation is of the form

$$
\begin{gathered}
\vdots \\
\frac{\Gamma, y: A \vdash_{G \oplus_{x}(x, y)} y: B, \Delta}{\Gamma \vdash_{G} x: A \supset B, \Delta} \supset R
\end{gathered}
$$

We prove $\left\langle\left\langle\Gamma \vdash_{G} x: A \supset B, \Delta\right\rangle\right\rangle_{x}$, which we know is enough by readdressing. The derivation is this:

$$
\begin{aligned}
& \vdots \text { IH on } \pi, y \\
& \frac{\frac{(\Lambda \vdash \Pi), A \vdash B}{(\Lambda \vdash \Pi) \vdash A \supset B}}{\Lambda \vdash A \supset B, \Pi} \text { unnest } L
\end{aligned}
$$

Here, $\Lambda \vdash \Pi=\left\langle\left\langle\Gamma \vdash_{G} \Delta\right\rangle\right\rangle_{x}$, which gives us $\left\langle\left\langle\Gamma \vdash_{G} x: A \supset B, \Delta\right\rangle\right\rangle_{x}=\Lambda \vdash A \supset B, \Pi$ and $\left\langle\left\langle\Gamma, y: A \vdash_{G \oplus_{x}(x, y)}\right.\right.$ $y: B, \Delta\rangle\rangle_{y}=(\Lambda \vdash \Pi), A \vdash B$. 


\subsection{From N-LBiI to L-LBiI}

The translation from N-LBiI to L-LBiI is intended as an inverse for that of the previous section. On sequents, it is a true inverse (translating a sequent from N-LBiI to L-LBiI and back, we arrive at exactly the same sequent; starting with an L-LBiI sequent, we get an isomorphic label tree with the same root). On derivations, the isomorphism should hold up to a suitable notion of equivalence of derivations on both sides (i.e., in both N-LBiI and L-LBiI). We will not pursue this here. But we expect that the right notions of equivalence would be best formulated and the isomorphism established with the help of term calculi.

We define a translation of N-LBiI sequents to $\mathbf{L}-\mathbf{L B i I}$ sequents, by induction on the antecedent and succedent of the given nested sequent, by the following function, which also takes a label $x$ as an additional argument. The root of the nesting structure of the given nested sequent (i.e., its top level) is sent to label $x$ in the label tree of the labelled sequent.

$$
\begin{aligned}
\llbracket \vdash \rrbracket_{x}= & \vdash_{\langle x\rangle} \\
\llbracket \vdash A, \Delta \rrbracket_{x}= & \Lambda \vdash_{G} x: A, \Pi \\
& \text { where } \Lambda \vdash_{G} \Pi=\llbracket \vdash \Delta \rrbracket_{x} \\
\llbracket \vdash\left(\Gamma_{0} \vdash \Delta_{0}\right), \Delta \rrbracket_{x}= & \Lambda, \Lambda_{0} \vdash_{G \oplus_{x}(x, y) \oplus_{y} G_{0}} \Pi_{0}, \Pi \\
\llbracket \Gamma, A \vdash \Delta \rrbracket_{x}= & \quad \text { where } \Lambda \vdash_{G} \Pi=\llbracket \vdash \Delta \rrbracket_{G} \text { and } \Lambda_{0} \vdash_{G_{0}} \Pi_{0}=\llbracket \Gamma_{0} \vdash \Delta_{0} \rrbracket_{y} \\
& \text { where } \Lambda \vdash_{G} \Pi=\llbracket \Gamma \vdash \Delta \rrbracket_{x} \\
\llbracket \Gamma,\left(\Gamma_{0} \vdash \Delta_{0}\right) \vdash \Delta \rrbracket_{x}= & \Lambda, \Lambda_{0} \vdash_{G_{0} \oplus_{y}(y, x) \oplus_{x} G} \Pi_{0}, \Pi \\
& \quad \text { where } \Lambda \vdash_{G} \Pi=\llbracket \Gamma \vdash \Delta \rrbracket_{x} \text { and } \Lambda_{0} \vdash_{G_{0}} \Pi_{0}=\llbracket \Gamma_{0} \vdash \Delta_{0} \rrbracket_{y}
\end{aligned}
$$

Intuitively, any formula in the top-level sequent is labelled by $x$ and remains where it is. Any sequent in the antecedent resp. succedent of the top-level sequent leads to the creation of a new label $y$ immediately below resp. above $x$. The translated elements of its antecedent resp. succedent are placed in the antecedent resp. succedent of the sequent in the making.

Note that we have given the mathematical definition by first recursing on the antecedent and then the succedent. In fact, the order is immaterial, one could just as well start with the antecedent or, indeed, remove formulas/nested sequents from the antecedent and succedent in turns, in any order. This commutativity is used extensively in our translation of derivations.

Theorem 3 If $\Gamma \vdash \Delta$ is derivable in $\mathbf{N}-\mathbf{L B i I}$, then $\llbracket \Gamma \vdash \Delta \rrbracket_{x}$ is derivable in L-LBiI for any $x$.

Proof By induction on the given derivation. We look at the following cases.

Case nestR: The given derivation is of the form

$$
\begin{gathered}
\vdots \\
\frac{\Gamma, \Gamma_{0} \vdash \Delta_{0}}{\Gamma \vdash\left(\Gamma_{0} \vdash \Delta_{0}\right), \Delta} \text { nestR }
\end{gathered}
$$


We can produce this derivation of the translated sequent:

$$
\begin{aligned}
& \vdots(\text { IH on } \pi)[y / x] \\
& \frac{\Lambda_{d}[y / x], \Lambda_{0} \vdash_{G_{d}[y / x] \oplus_{y} G_{0}} \Pi_{0}, \Pi_{d}[y / x]}{\Lambda_{d}[y / x], \Lambda_{0} \vdash_{G_{d} \oplus_{x}(x, y) \oplus_{y} G_{0}} \Pi_{0}, \Pi_{d}[y / x]} \text { nodesplitD }
\end{aligned}
$$

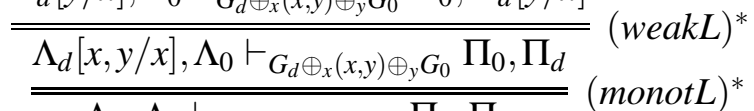

$$
\begin{aligned}
& \frac{\Lambda_{d}, \Lambda_{0} \vdash_{G_{d} \oplus_{x}(x, y) \oplus_{y} G_{0}} \Pi_{0}, \Pi_{d}}{\overline{\Lambda_{d}, \Lambda_{0} \vdash_{G_{d}}}}(\text { monot } L)^{*}
\end{aligned}
$$

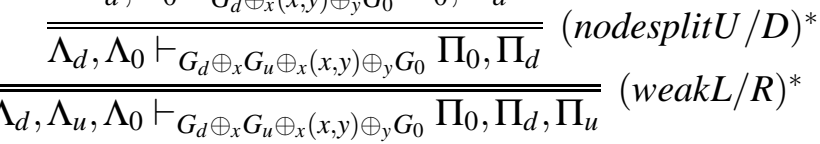

where $\Lambda_{d} \vdash_{G_{d}} \Pi_{d}=\llbracket \Gamma \vdash \rrbracket_{x}, \Lambda_{u} \vdash_{G_{u}} \Pi_{u}=\llbracket \vdash \Delta \rrbracket_{x}$ and $\Lambda_{0} \vdash_{G_{0}} \Pi_{0}=\llbracket \Gamma_{0} \vdash \Delta_{0} \rrbracket_{y}$, and $\Lambda_{d}[x, y / x]$ stands for the union of $\Lambda_{d}[y / x]$ with the context formed by the $x$-labelled formulas of $\Lambda_{d}$. Notice that $x \notin\left|\Pi_{d}\right|$, which tells us that $\Pi_{d}[y / x]=\Pi_{d}$. The side condition of the topmost application of nodesplitD is met because $G_{d} \uparrow x$. Note also that particular cases of nodesplit $U / D$ allow the addition of new nodes to a label tree.

Case unnest $L$ : We are given a derivation in the form

$$
\begin{gathered}
\vdots \\
\frac{\Gamma,\left(\Gamma_{0} \vdash \Delta_{0}\right) \vdash \Delta}{\Gamma, \Gamma_{0} \vdash \Delta_{0}, \Delta} \text { unnest } L
\end{gathered}
$$

We make the derivation

$$
\begin{gathered}
\vdots \text { IH on } \pi \\
\frac{\Lambda, \Lambda_{0}[y / x] \vdash_{G_{0}[y / x] \oplus_{y}(y, x) \oplus_{x} G} \Pi_{0}[y / x], \Pi}{\Lambda, \Lambda_{0} \vdash_{G_{0} \oplus_{x} G} \Pi_{0}, \Pi} \text { nodemergeD }
\end{gathered}
$$

where $\Lambda \vdash_{G} \Pi=\llbracket \Gamma \vdash \Delta \rrbracket_{x}$ and $\Lambda_{0} \vdash_{G_{0}} \Pi_{0}=\llbracket \Gamma_{0} \vdash \Delta_{0} \rrbracket_{x}$.

Case $\supset R$ : The given derivation is of the form

$$
\begin{gathered}
\vdots \\
\frac{\Gamma, A \vdash B}{\Gamma \vdash A \supset B, \Delta} \supset R
\end{gathered}
$$

We transform it to

$$
\begin{aligned}
& \vdots(\text { IH on } \pi)[y / x] \\
& \underline{\frac{\Lambda_{d}[y / x], y: A \vdash_{G_{d}[y / x]} y: B, \Pi_{d}[y / x]}{\Lambda_{d}[y / x], y: A \vdash_{G_{d} \oplus_{x}(x, y)} y: B, \Pi_{d}[y / x]}} \text { nodesplitD } \\
& \frac{\frac{\Lambda_{d}[x, y / x], y: A \vdash_{G_{d} \oplus_{x}(x, y)} y: B, \Pi_{d}}{\frac{\Lambda_{d}, y: A \vdash_{G_{d} \oplus_{x}(x, y)} y: B, \Pi_{d}}{\Lambda_{d} \vdash_{G_{d}} x: A} \supset B, \Pi_{d}}(\text { monot } L)^{*}}{}(\text { weakL })^{*} \\
& \frac{\Lambda_{d}, y: A \vdash_{G_{d} \oplus_{x}(x, y)} y: B, \Pi_{d}}{\Lambda_{d} \vdash_{G_{d}} x: A \supset B, \Pi_{d}} \supset R \\
& \frac{\frac{\Lambda_{d} \vdash_{G_{d} \oplus_{x} G_{u}} x: A \supset B, \Pi_{d}}{\overline{\Lambda_{d}, \Lambda_{u} \vdash_{G_{d} \oplus_{x} G_{u}} x: A \supset B, \Pi_{d}, \Pi_{u}}}(\text { nodesplit } / D)^{*}}{\text { weakL } / R)^{*}}
\end{aligned}
$$

where $\Lambda_{d} \vdash_{G_{d}} \Pi_{d}=\llbracket \Gamma \vdash \rrbracket_{x}$ and $\Lambda_{u} \vdash_{G_{u}} \Pi_{u}=\llbracket \vdash \Delta \rrbracket_{x}$. Notice that $x \notin\left|\Pi_{d}\right|$, with the effect that $\Pi_{d}[y / x]=$ $\Pi_{d}$. The side condition on the topmost application of nodesplitD is satisfied as $G_{d} \uparrow x$. 


\subsection{From LBiI into L-LBiI and back}

The translation of $\mathbf{L B i I}$ into $\mathbf{L}-\mathbf{L B i I}$ is not demanding. Essentially, it suffices to annotate the end sequent with the sole label of a singleton label tree and follow the structure of the LBiI-derivation bottom-up, introducing new labels at $\supset R$ and $\prec L$. But again (like in the translation from LBiI to N-LBiI), a cut in $\mathbf{L B i I}$ is rendered by a cut in $\mathbf{L - L B i I}$, which is not so perfect, since we should not need cut in $\mathbf{L}-\mathbf{L B i I}$ derivations.

When we wrote the translation, we did not think of it like this, but it can be described as composition of the translations from $\mathbf{L B i I}$ to $\mathbf{N}$-LBiI and from N-LBiI further on to L-LBiI. Since LBiI sequents yield no nesting in $\mathbf{N}$-LBiI, the readdressing that is needed in translating derivations has only to do with the $\supset R$ and $\prec L$ rules.

Given a standard context $\Gamma$, we write $x: \Gamma$ for the labelled context obtained by labelling all formulas of $\Gamma$ with $x$.

Theorem 4 If $\Gamma \vdash \Delta$ is derivable in LBiI, then $x: \Gamma \vdash_{\langle x\rangle} x: \Delta$ is derivable in L-LBiI.

Proof By induction on the derivation of $\Gamma \vdash \Delta$ in LBiI. We show one case.

Case $\supset R$ : The given derivation

$$
\begin{gathered}
\vdots \\
\frac{\Gamma, A \vdash B}{\Gamma \vdash A \supset B, \Delta} \supset R
\end{gathered}
$$

is matched with the derivation

$$
\begin{gathered}
\vdots \\
\text { IH on } \pi \\
\frac{\frac{y: \Gamma, y: A \vdash_{\langle y\rangle} y: B}{y: \Gamma, y: A \vdash_{(x, y)} y: B}}{\frac{x: \Gamma, y: \Gamma, y: A \vdash_{(x, y)} y: B}{x: \Gamma, y: A \vdash_{(x, y)} y: B}} \text { wedesplitD } \\
\text { monot } L \\
\frac{x: \Gamma \vdash_{\langle x\rangle} x: A \supset B}{x: \Gamma \vdash_{\langle x\rangle} x: A \supset B, x: \Delta} \\
\text { (weakR })^{*}
\end{gathered}
$$

The translation from $\mathbf{L}-\mathbf{L B i I}$ to $\mathbf{L B i I}$ is best found by as a compound translation through $\mathbf{N}-\mathbf{L B i I}$. We omit the details here, but it is quite instructive. In particular, it gives a kind of explanation of why it is so difficult to translate labelled derivations into standard derivations in the case of Int. We learn that the natural way uses exclusion, and this is not available in Int.

\section{Applications of the translations}

By analysing the targets of the various translations, one can find some immediate applications. Our analysis essentially focuses on how much cuts are needed in the translations, thus finding complete classes of cuts. A direct use of the translations, not explored here, is as a means of mapping proofs found by known search procedures for BiInt, based on the nested calculus and on the labelled calculus, back into standard-style sequent calculus. 


\section{Translation from N-LBiI into LBiI}

In this translation, the cut rule of $\mathbf{L B i I}$ is used only for the translation of the cut rule of $\mathbf{N}$-LBiI and of the unnesting-rules. Let us call unnest cuts the cuts of LBiI of one of the following two forms:

$$
\begin{aligned}
& \frac{\Gamma, \Gamma_{0} \vdash \wedge \Gamma_{0} \prec \bigvee \Delta_{0}, \Delta_{0}, \Delta \quad \Gamma, \Gamma_{0}, \wedge \Gamma_{0} \prec \bigvee \Delta_{0} \vdash \Delta_{0}, \Delta}{\Gamma, \Gamma_{0} \vdash \Delta_{0}, \Delta} \text { unnestcutL } \\
& \frac{\Gamma, \Gamma_{0} \vdash \bigwedge \Gamma_{0} \supset \bigvee \Delta_{0}, \Delta_{0}, \Delta \quad \Gamma, \Gamma_{0}, \wedge \Gamma_{0} \supset \bigvee \Delta_{0} \vdash \Delta_{0}, \Delta}{\Gamma, \Gamma_{0} \vdash \Delta, \Delta_{0}} \text { unnestcutR }
\end{aligned}
$$

Observe that these two special cases of cut are the ones used in the translations of the unnest rules. As N-LBiI is complete without cut, we have that unnest cuts are complete for $\mathbf{L B i I}$.

Proposition 1 The sequent calculus obtained from $\mathbf{L B i I}$ by restricting to unnest cuts is complete for BiInt.

Now of course the first premise of unnestcut $L$ and the second premise of unnestcut $R$ are derivable, so a more practical idea would be to remove cuts altogether and instead make the rules

$$
\frac{\Gamma, \Gamma_{0}, \bigwedge \Gamma_{0} \prec \bigvee \Delta_{0} \vdash \Delta_{0}, \Delta}{\Gamma, \Gamma_{0} \vdash \Delta_{0}, \Delta} \text { unnest } L \quad \frac{\Gamma, \Gamma_{0} \vdash \wedge \Gamma_{0} \supset \bigvee \Delta_{0}, \Delta_{0}, \Delta}{\Gamma, \Gamma_{0} \vdash \Delta_{0}, \Delta} \text { unnest } R
$$

\section{From N-LBiI to LBiI and back and then there again}

The attempt at a direct cut elimination transformation for LBiI fails because of three combinations of cuts with other rules [9]. They fall into two wider combinations:

$$
\frac{\Gamma \vdash A, C \supset D, \Delta \quad \frac{\Gamma, A, C \vdash D}{\Gamma, A \vdash C \supset D, \Delta}}{\Gamma \vdash C \supset D, \Delta} \text { cut } \quad \frac{\frac{C \vdash D, A, \Delta}{\Gamma, C \prec D \vdash A, \Delta} \prec L \quad \Gamma, C \prec D, A \vdash \Delta}{\Gamma, C \prec D \vdash \Delta} \text { cut }
$$

The unnest $L$ and unnest $R$ rules give a possibility to permute the cuts up past the $\supset R$ and $\prec L$ inferences in these two configurations.

We show this for the $\supset R$ case:

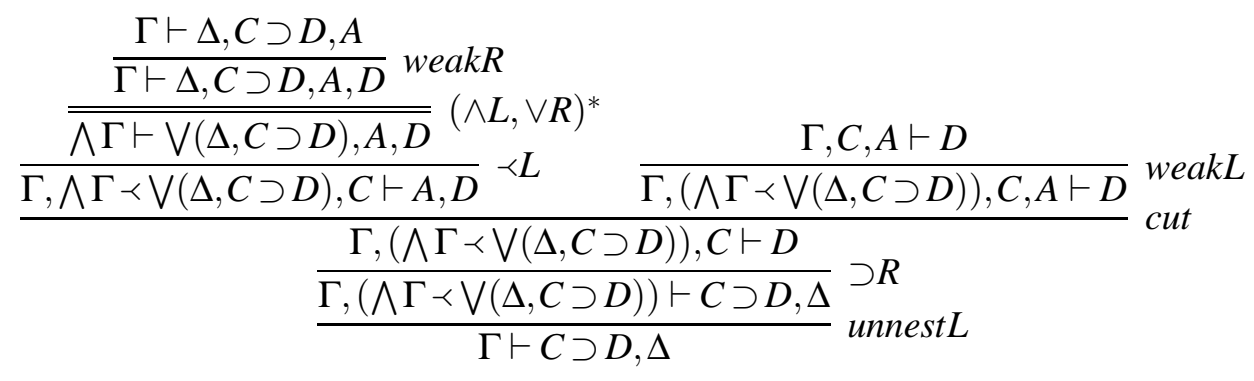

The possibility of this permutation may give a transformation replacing the cuts in a LBiI derivation with unnest $L$ and unnest $R$, but we have not checked if it is welldefined, i.e., terminates.

\section{Translations between L-LBiI into N-LBiI}

In these translations, cuts in the target are only needed for translating cuts in the source. Thus, redundancy

of cut in one implies redundancy of cut in the other. 


\section{Final remarks}

Our translations between standard-style, nested and labelled sequent calculi for BiInt provide a framework for comparison of proof transformations within each of these systems. A basic question is to understand the relationship between ways of performing cut-elimination in the three systems: (i) for the nested system, Goré et al. [7] have described a cut-elimination procedure; (ii) for the labelled system, one should be able to adapt Negri's procedure [10], which applies to a wide range of normal modal logics; (iii) for the standard-style system, cuts are not fully eliminable, but unnest cuts are a complete and simple form of cuts.

A tool that should be helpful to perform comparison of cut-elimination processes is term assignment. We are not aware of term assignment done directly for systems based on nested sequents. This kind of formalisms have been used mostly in connection with proof search and modal logics, exploiting the subformula property [2], but for BiInt, besides the study of shallow inference and nested sequents of Goré et al. [7], a study of deep inference and nested sequents (though still with emphasis on proof search) is also available [12]. As to labelled systems, Reed and Pfenning [16] consider term assignment in the context of labelled intuitionistic logic. They work with natural deduction and use control operators letcc and throw to account for the multiple conclusions of the labelled sequent calculus that they proceed from. A term assignment for BiInt corresponding to Dragalin's style can be obtained from the wellstudied calculus $\bar{\lambda} \mu \tilde{\mu}$ with the typing system $L K_{\mu \tilde{\mu}}$ of Curien-Herbelin [6]. From these systems, which are for classical logic (or for classical logic with exclusion [6]), we obtain the $\supset, \prec$-fragment of BiInt by imposing the usual single formula restriction in the succedent (resp. antecedent) of the rule corresponding to $\supset R$ (resp. $\prec L$ ), i.e.,

$$
\frac{\Gamma, x: A \vdash v: B \mid}{\Gamma \vdash \lambda x . v: A \supset B \mid \Delta} \supset R \quad \frac{\mid e: B \vdash \beta: A, \Delta}{\Gamma \mid \beta \lambda . e: B \prec A \vdash \Delta} \prec L
$$

The exclusion operation has been given computational meaning by, e.g., Crolard [5] and Ariola et al. [1]. Crolard considered multiple-conclusion natural deduction systems both for classical logic with exclusion and for BiInt, the latter being obtained from the former by a mechanism to keep track of dependencies between hypotheses and conclusions and ensure the BiInt-restrictions, arriving at a safe $\lambda$-calculus, where coroutines (a restricted form of continuations) become first-class objects. Ariola et al. considered classical logic with exclusion in a natural deduction system close to that of Crolard's, to provide a typing system for a $\lambda$-calculus with delimited continuations. It would be interesting to revisit these ideas in connection to the sequent calculi studied in this paper and understand whether the sequent calculus format (standard or extended) has anything new to offer. As Crolard's mechanism to keep track of dependencies resembles our labelled system for BiInt, a specific goal would be to investigate relationships between the two systems.

Acknowledgments We are grateful to our anonymous referees for their helpful comments. We are also grateful to Linda Postniece for discussions. We were supported by the Portuguese Foundation for Science and Technology through Centro de Matemática da Universidade do Minho and project RESCUE no. PTDC/EIA/65862/2006, ERDF through the Estonian Centre of Excellence in Computer Science, EXCS, and the Estonian Science Foundation under grant no. 6940. 


\section{References}

[1] Z. M. Ariola, H. Herbelin \& A. Sabry (2009): A type-theoretic foundation of delimited continuations. Higher-Order and Symbolic Computation 22(3), pp. 233-273. Available at http://dx.doi.org/10.1007/s10990-007-9006-0.

[2] K. Brünnler (2010). Nested Sequents. Habilitation thesis. Available at http://arxiv.org/abs/1004.1845

[3] L. Buisman (Postniece) \& R. Goré (2007): A cut-free sequent calculus for bi-intuitionistic logic. In: N. Olivetti, editor: Proc. of 16th Int. Conf. on Automated Reasoning with Analytic Tableaux and Related Methods, TABLEAUX 2007 (Aix en Provence, July 2007), Lect. Notes in Comput. Sci. 4548, Springer, pp. 90-106. Available at http://dx.doi.org/10.1007/978-3-540-73099-6_9

[4] T. Crolard (2001): Subtractive logic. Theor. Comput. Sci. 254(1-2), pp. 151-185. Available at http://dx.doi.org/10.1016/S0304-3975(99)00124-3.

[5] T. Crolard (2004): A formulae-as-types interpretation of subtractive logic. J. of Log. and Comput. 14(4), pp. 529-570. Available at http://dx.doi.org/10.1093/logcom/14.4.529

[6] P.-L. Curien \& H. Herbelin (2000): The duality of computation. In: Proc. of 5th Int. Conf. on Functional Programming, ICFP 'O0 (Montreal, Sept. 2000), ACM Press, pp. 233-243. Available at http://doi.acm.org/10.1145/351240.351262.

[7] R. Goré, L. Postniece \& A. Tiu (2008): Cut-elimination and proof-search for bi-intuitionistic logic using nested sequents. In: C. Areces \& R. Goldblatt, editors: Advances in Modal Logic, 7, College Publications, pp. 43-66. Available at http://www.aiml.net/volumes/volume7/Gore-Postniece-Tiu.pdf.

[8] P. Łukowski (1996): Modal interpretation of Heyting-Brouwer logic. Bull. of Sect. of Logic 25(2), pp. 80-83. Available at http://www.filozof.uni.lodz.pl/bulletin/pdf/25_2_3.pdf.

[9] C. Monteiro (2006): Caracterizações semânticas e dedutivas da lógica bi-intuicionista. Master's thesis, Universidade de Trás-os-Montes e Alto-Douro.

[10] S. Negri (2005): Proof analysis in modal logic. J. of Philos. Logic 34(5-6), pp. 507-544. Available at http://dx.doi.org/10.1007/s10992-005-2267-3.

[11] L. Pinto \& T. Uustalu (2009): Proof search and counter-model construction for bi-intuitionistic propositional logic with labelled sequents. In: M. Giese \& A. Waaler, editors: Proc. of 18th Int. Conf. on Automated Reasoning with Analytic Tableaux and Related Methods, TABLEAUX 2009 (Oslo, July 2009), Lect. Notes in Artif. Intell. 5607, Springer, pp. 295-309. Available at http://dx.doi.org/10.1007/978-3-642-02716-1_22

[12] L. Postniece (2009): Deep Inference in Bi-intuitionistic Logic. In: H. Ono, M. Kanazawa \& R. Queiroz, editors: Proc. of 16th Int. Wksh. on Logic, Language, Information and Computation, WoLLiC 2009 (Tokyo, June 2009), Lect. Notes in Artif. Intell. 5514, Springer, pp. 320-334. Available at http://dx.doi.org/10.1007/978-3-642-02261-6_26.

[13] C. Rauszer (1974): A formalization of the propositional calculus of H-B logic. Studia Logica 33(1), pp. 23-34. Available at http://dx.doi.org/10.1007/bf02120864.

[14] C. Rauszer (1974): Semi-boolean algebras and their applications to intuitionistic logic with dual operators. Fund. Math. 83, pp. 219-249. Available at http://matwbn.icm.edu.pl/ksiazki/fm/fm83/fm83120.pdf.

[15] C. Rauszer (1977): Applications of Kripke models to Heyting-Brouwer logic. Studia Logica 36(1-2), pp. 61-71. Available at http://dx.doi.org/10.1007/bf02121115.

[16] J. Reed \& F. Pfenning (2009): Intuitionistic letcc via labelled deduction. Electron. Notes in Theor. Comput. Sci. 231, pp. 91-111. Available at http://dx.doi.org/10.1016/j.entcs.2009.02.031.

[17] G. Restall (1977). Extending intuitionistic logic with subtraction. Unpublished note. Available at http://consequently.org/writing/extendingj/. 